\title{
Autologous stem cell transplantation in elderly patients with multiple myeloma: evaluation of its safety and efficacy.
}

\author{
Original Article
}

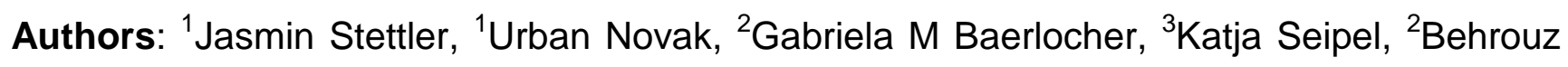
Taleghani Mansouri, ${ }^{1}$ Thomas Pabst.

Affiliations: 'Department of Medical Oncology, Inselspital, University Hospital Bern; ${ }^{2}$ Department of Hematology, Inselspital, University Hospital Bern; and ${ }^{3}$ Department of Clinical Research, University of Bern, Switzerland.

Authorship and contribution: J.S.: performed research, analyzed data and wrote the paper; U.N.: analyzed data and reviewed the manuscript; G.M.B.: contributed relevant data and material and reviewed the manuscript; K.S.: analyzed data, contributed relevant data and reviewed the manuscript; B.M.T.: contributed relevant data and material and reviewed the manuscript; T.P.: designed research, analyzed data, and wrote the paper.

Key words: myeloma; elderly; high-dose chemotherapy; autologous; transplant; stem cell; survival; prognosis; safety; efficacy.

Manuscript information: total: 18 pages; 2656 words (allowed 3'000); abstract: 150 words (allowed 150); 39 references; 3 tables; 1 figure.

Running title: Autologous transplant in elderly myeloma patients.

Conflict of interests: All authors declare no conflict of interest.

Funding: This work was supported by grants from the Krebsliga Bern (to TP), and from the EMPIRIS Foundation/Ursula-Hecht-Fonds (to TP).

Corresponding author: Thomas Pabst M.D.; Associate Professor; Department of Medical Oncology; University Hospital; 3010 Bern; Switzerland. Phone +41 31632 8430; Fax +41 31 632 3410; Email: thomas.pabst@insel.ch. 


\section{ABSTRACT}

Administering high-dose chemotherapy (HDCT) with melphalan to elderly myeloma patients represents a challenge with respect to achieving therapeutic efficacy whilst avoiding significant toxicity. We analyzed safety and efficacy of HDCT in 61 elderly myeloma patients older than 65 years, including 12 patients $\geq 70$ years, and compared them to $237 \mathrm{MM}$ patients below 65 years treated in the same period. MM patients older than 70 years had a longer duration of hospitalization (26 vs. 20 days; $P=.0001)$ and a longer time until platelet recovery $>20$ G/L (19 vs. 15 days; $P=.0398)$. However, we observed no differences in the time until neutrophil recovery, infection rate, and treatment related mortality until 100 days after ASCT. Furthermore, higher age was not associated with inferior progression-free and overall survival at 1 and 2 years after ASCT. Our data suggest that HDCT with ASCT is feasible, safe and effective in MM patients older than 65 years. 


\section{INTRODUCTION}

Symptomatic multiple myeloma $(\mathrm{MM})$ is a malignant plasma cell disease characterized by the presence of a specific monoclonal immunoglobulin and related organ damages including hypercalcemia, renal insufficiency, anemia, or lytic bone lesions (CRAB) [1,2]. Most symptomatic MM patients are elderly, defined as being older than 65 years of age, with the incidence of MM increasing along the aging of the general population and its increasing life expectancy.

Since the late 1990s, high-dose melphalan treatment $(200 \mathrm{mg} / \mathrm{m} 2)$ followed by autologous stem cell transplantation (ASCT) has been regarded as a standard procedure for younger MM patients in the absence of relevant comorbidities [3-5]. In the first decade of the 21st century, bortezomib-based regimens have become standard induction therapy preceding ASCT in transplant-eligible patients and have additionally contributed to improved survival for younger MM patients [6-10]. However, the significant improvement in overall survival has mainly been observed in younger MM patients [11-17], whereas advances for elderly MM patients have been less significant $[11,14,16,18]$.

MM patients aged older than 65 years are usually considered as ineligible for highdose melphalan therapy $(200 \mathrm{mg} / \mathrm{m} 2)$ followed by ASCT mainly because of impairment in organ functions and reduced drug metabolisms. Consequently, clinical trials involving ASCT have mostly been restricted to patients younger than 65 years with few exceptions. The toxicity of $200 \mathrm{mg} / \mathrm{m} 2$ high-dose melphalan was investigated in 25 elderly patients aged 70 years or older [19] resulting in four (16\%) transplant-related deaths (TRM), but after reducing the dose to $140 \mathrm{mg} / \mathrm{m} 2$ the TRM could be reduced to $2 \%$ while maintaining the efficacy. Of crucial importance was a 
subsequent trial with $2-3$ courses of ASCT with $100 \mathrm{mg} / \mathrm{m} 2$ of melphalan in 71 patients aged up to 75 years [20]. When compared with matched-pair patients treated with melphalan and prednison (MP), the efficacy of ASCT was significantly superior to MP in terms of complete response (CR) rate, event-free survival (EFS) and OS, without transplant-related deaths. Based on these reports, elderly patients with $\mathrm{MM}$ in otherwise fit medical condition have been considered to be eligible for ASCT irrespective of the chronological age in many institutions [21,22]. Accordingly, several single institution studies have reported their experience of ASCT in elderly patients [23-30]. As conditioning regimen before ASCT, reduced dosing of melphalan (100-140mg/m2) has been predominantly used in elderly patients, with no significant difference in transplant-related mortality and morbidity between elderly and younger patients. Recently, the transplant-related mortality in MM patients has decreased to $2-4 \%$, probably due to the improvement of supportive therapies [31]. As a consequence, reduced-intensity ASCT is considered to be a safe and effective therapeutic modality even in MM patients aged 65-75 years in a good performance status without relevant comorbidities. Accordingly, physicians increasingly tend to raise the upper age limit, and a substantial number of elderly MM patients is offered ASCT in clinical routine nowadays.

In order to further evaluate the safety and efficacy of ASCT in this cohort of patients, we performed a retrospective analysis of data obtained from elderly MM patients aged older than 65 years at our center. We compared these results with data obtained from MM patients younger than 66 years of age treated in the same period to assess the toxicity and the survival benefit of HDCT with ASCT in elderly MM patients. 


\section{MATERIAL AND METHODS}

Patients: Patients with MM consecutively seen at the University Hospital of Bern, Switzerland from January 2009 to May 2015 who received at least one HDCT with ASCT were studied. All patients had given written informed consent, and this study was approved by the local ethics committee of Bern, Switzerland (decision number $\# 149$-14). The patients were stratified into three groups according to their age at ASCT. Group 1 comprised MM patients aged 18-65 years, group 2 involved MM patients aged 66-69 years, and MM patients in group 3 were $\geq 70$ years.

\section{$\underline{\text { Treatment }}$}

According to local standards, non-myelosuppressive chemotherapy with vinorelbine or gemcitabine, but not cyclophosphamide, together with G-CSF was used for mobilization of autologous stem and progenitor cells [32-34]. At least $3 \times 10^{6}$ CD34+ cells/kg body weight (b.w.) were collected per planned stem cell transplantation. Patients above 70 years received a reduced dose of melphalan $(140 \mathrm{mg} / \mathrm{m} 2)$, whereas patients $\leq 70$ years were administered full dose melphalan $(200 \mathrm{mg} / \mathrm{m} 2)$; the dose of melphalan was reduced to $140 \mathrm{mg} / \mathrm{m} 2$ for patients with reduced renal function with a creatinine-clearance between $40-50 \mathrm{ml} / \mathrm{min}$, and to $100 \mathrm{mg} / \mathrm{m} 2$ for patients with a creatinine-clearance below $40 \mathrm{ml} / \mathrm{min}$. All patients received weight adapted G-CSF starting at day 6 after ASCT until neutrophils exceeded $0.5 \mathrm{G} / \mathrm{L}$ for three consecutive days. Patients routinely had antiviral (acyclovir 500mg twice daily) and antifungal prophylaxis (fluconazole 400mg once weekly and sulfamethoxazol/trimethoprim 800/160mg three times per week). 


\section{Definitions}

Objectives of the study with regards to safety were duration of hospitalization, time until neutrophil and platelet recovery, rate of febrile episodes, and mortality until 100 days after ASCT, as well as the occurrence of unexpected toxicities during and after hospitalization for ASCT until 100 days after ASCT. Neutropenia was defined as neutrophil counts $<0.5 \mathrm{G} / \mathrm{l}$, and thrombocytopenia as platelet counts $<20 \mathrm{G} / \mathrm{l}$. The mortality until day 100 was defined as death from any cause within 100 days after ASCT, and TRM was defined as transplant-related death due to toxicity or infectious complications following ASCT.

OS was defined as the time from ASCT until death from any cause or last follow-up. PFS was defined as the time from ASCT until first relapse/progression, death or last follow-up, whichever occurred first. Complete remission (CR) was defined as a negative immunofixation in serum and urine, absence of plasmocytoma and less than $5 \%$ plasma cells in the bone marrow. A reduction of $\geq 50 \%$ of serum M-protein was considered a partial response (PR). Progression (PD) was defined as an increase of at least $25 \%$ in measurable monoclonal immunoglobulin in serum or urine or an increase of $\geq 25 \%$ in urinary light chains. If $\mathrm{CR}$ lasted less than 3 months, it was defined as progression (PD).

\section{$\underline{\text { Statistical Analysis }}$}

Survival curves were calculated using the method of Kaplan and Meier and compared using the log-rank test. Differences in the mean values of continuous variables were tested using the t-test. All statistical analyses and graphs were performed using graph pad prism program 7.00 (Trial Version) or Microsoft Excel (Microsoft Office 2016). P-values <0.05 were considered statistically significant. 


\section{RESULTS}

We identified a cohort of 298 patients with symptomatic multiple myeloma (MM) who received at least one high-dose chemotherapy (HDCT) with melphalan with autologous stem cell transplantation (ASCT) between January 2009 and May 2015 at the University Hospital Bern, Switzerland. The patients were divided into three age groups: Group 1 comprised MM patients aged 18-65 years, group 2 involved MM patients aged 66-69 years, and MM patients in group 3 were $\geq 70$ years. The patient characteristics at diagnosis are summarized in Table I. We observed that elderly MM patients (group 2) had more frequently advanced disease (ISS stage III) than younger MM patients in group 1 (59\% vs. 39\%; $P=.0073)$, respectively. The Supplemental Table I depicts the mobilization and high dose chemotherapy regimens used in this study.

Table II depicts the details on mobilization and collection of autologous stem and progenitor cells. All MM patients were mobilized with a single dose of nonmyelosuppressive chemotherapy using $35 \mathrm{mg} / \mathrm{m} 2$ of vinorelbine or $1250 \mathrm{mg} / \mathrm{m} 2$ of gemcitabine (but not cyclophosphamide) together with G-CSF [32-34], with a collection goal of at least $3 \times 10^{6}$ CD34+ cells $/ \mathrm{kg}$ body weight (b.w.) per planned stem cell transplantation. Apheresis was initiated based on pre-collection peripheral CD34+ cell counts, and a minimum of 15 peripheral CD34+ cells/ $\mu$ l was necessary to trigger the collection procedure. The minimal number of collected CD34+ cells to proceed to transplantation was $2 \times 10^{6} / \mathrm{kg}$ b.w. 
254 MM patients (85\%) achieved the pre-defined collection goal in a single apheresis day procedure, whereas 44 patients (15\%) needed two days. Whereas we observed no differences between the three age groups in the number of apheresis days needed (two days of apheresis in 14\%, 20\%, and 17\% in groups 1, 2 and 3, respectively, $P=.455$ ), we found that elderly patients mobilized significantly less circulating peripheral CD34+ cells at the day of stem cell collection $\left(110 \times 10^{6} / \mathrm{l}\right.$, $77 \times 10^{6} / \mathrm{l}$ and $50 \times 10^{6} / \mathrm{I}$ in groups 1,2 and 3 , respectively, $P=.0097$ ). Also, the percentage of circulating CD34+ cells per peripheral leukocytes at the day of stem cell collection was lower in elderly MM patients $(0.5 \%, 0.29 \%$, and $0.26 \%$ in groups 1, 2 and 3, respectively, $P=.2914)$. Due to a longer duration of stem cell collection in elderly patients (details not shown), the total number of collected CD34+ cells was comparable in all three age groups. Accordingly, the total number of re-transfused autologous stem cells was similar in all three age groups (Table II).

All MM patients in this cohort received melphalan conditioning as planned: Patients above 70 years were given a reduced dose of melphalan $140 \mathrm{mg} / \mathrm{m} 2$, whereas melphalan $200 \mathrm{mg} / \mathrm{m} 2$ was administered in patients younger than 70 years. The melphalan dose was reduced to $140 \mathrm{mg} / \mathrm{m} 2$ for patients with reduced renal function with a creatinine-clearance between $40-50 \mathrm{ml} / \mathrm{min}$, and to $100 \mathrm{mg} / \mathrm{m} 2$ for patients with a creatinine-clearance of below $40 \mathrm{ml} / \mathrm{min}$.

We observed that age $\geq 70$ years (group 3) was associated with prolonged hospitalization for HDCT with ASCT compared to the age groups 1 and 2 (26 days in group 3 vs. 19 and 20 days in groups 1 and 2; $P=.0 .001)$. We also found differences for the time needed until hematologic recovery (Table III). Age $\geq 70$ years (group 3) was associated with a prolonged duration until recovery of platelets above $20 \mathrm{G} / \mathrm{L}$ (20 days in group 3 vs. 13 days in groups 1 and 2; $P=.007)$. In contrast, no differences 
were seen between the three age groups in the duration of neutropenia $(12,11$ and 11 days in groups 1,2 and $3, P=.0751$ ).

A total of 44 patients have died since ASCT (15\%). Four (1\%) patients - all aged below 66 years - died within 100 days after ASCT, and all four (1\%) deaths were due to transplant-related causes (TRM) comprising one patient dying because of septic shock and three patients dying because of respiratory tract infections with respiratory failure (Table III). TRM was not different among the three age cohorts (2\% in group 1 and $0 \%$ in groups 2 and $3 ; P=.5935)$.

The number of febrile episodes and the number of febrile episodes with identification of a causing germ was similar between the three age groups, both during hospitalization as well as after discharge until 100 days after ASCT. Similarly, no differences were observed for the rate of severe complications and/or additional hospitalizations after discharge from HDCT/ASCT treatment between the three cohorts (13\%, 22\%, and 17\%; $P=.2016)$.

Before ASCT, the rate of patients in CR after induction treatment was comparable between the three age groups (Supplemental Table II). Similarly, we observed no differences in the rate of achieved complete remissions (CR1) 100 days after ASCT (47\%, 45\%, and $58 \%$ in groups 1,2 and $3 ; P=.7018)$. After a mean follow-up of 21 months after ASCT, 114 myeloma patients (38\%) in our cohort had a relapse, and the relapse rate was not higher in older patients $(42 \%, 20 \%$, and $33 \% ; P=.0151)$, and the median duration until first relapse was similar in all three age groups $(22,18$ and 8 months in group 1,2 and 3; $P=.121$ ). We observed no differences in the PFS between the three age groups (Figure 1). 
Finally, 44 deaths (15\%) were reported in our cohort, with age above 65 years not being associated with increased death rate $(18 \%, 2 \%$, and $0 \%$ in group 1, 2 and 3 ; $P=.0052)$. The OS was not inferior for elderly myeloma patients compared to patients below 66 years (Figure 1 and Supplemental Table II). As expected, the failure to achieve CR within 100 days after ASCT was associated with a less favorable PFS and OS ( $P=.0001)$ (Supplemental Figure 1A and 1B). For patients achieving a CR after ASCT, PFS and OS for patients above 65 years were comparable to younger myeloma patients (Supplemental Figure 1C and 1D). These data suggest that elderly fit myeloma patients experience similar survival benefits from HDCT with ASCT as myeloma patients below 66 years. 


\section{DISCUSSION}

Few studies are available which incorporated consolidation with ASCT into the treatment algorithm for elderly symptomatic myeloma patients in the era of novel agents. Two phase II trials have consolidated the induction treatment - with four cycles of bortezomib, pegylated liposomal doxorubicin and dexamethasone - with tandem ASCT (each $100 \mathrm{mg} / \mathrm{m} 2$ of melphalan), followed by consolidation therapy with four cycles of lenalidomide and prednisone, and a maintenance therapy with lenalidomide alone in patients aged $65-75$ years $[35,36]$. The median PFS was 48 months, and the 5-year overall survival (OS) was 63\%; notably, patients achieving a CR had a 5 -year OS of $83 \%$. The treatment-related mortality associated with ASCT in these two trials was significantly higher in MM patients $\geq 70$ years (19\%) compared with that of MM patients (4\%) less than 70 years $(P=0.024)$; thus, one might conclude that a sequential approach including reduced-intensity ASCT may benefit patients younger than 70 years of age with good performance status and without comorbidities [37-39]. In line with these reports we found in our retrospective singlecenter cohort not a single treatment-related death during the 100 days after ASCT in 71 MM patients aged between 66 and 69 years. However, we also observed no treatment-related death in a (small) cohort of 20 myeloma patients aged $\geq 70$ years at ASCT. Our data therefore point to the possibility that age adapted high-dose melphalan with ASCT can be safely applied to consolidate induction treatment with novel agents in elderly fit myeloma patients, including myeloma patients above the age of 70 years.

Importantly, we followed a strategy of applying $200 \mathrm{mg} / \mathrm{m} 2$ melphalan before ASCT in myeloma patients below 70 years, and we reduced melphalan to $140 \mathrm{mg} / \mathrm{m} 2$ for 
patients $\geq 70$ years. In addition, dose reductions were performed for patients with reduced renal function. The transplant-related mortality (TRM) for melphalan 200 $\mathrm{mg} / \mathrm{m} 2$ in elderly myeloma patients was reported to be up to $19 \%(17)$, while that for $100 \mathrm{mg} / \mathrm{m} 2$ of melphalan was $0 \%$ (19) and $2 \%$ for $140 \mathrm{mg} / \mathrm{m} 2$ melphalan (20). Our single-center results with a TRM of $0 \%$ in 71 myeloma patients aged 66-69 years treated with $200 \mathrm{mg} / \mathrm{m} 2$ melphalan challenge the need for dose reductions in myeloma patients in this age cohort considered to be fit for high-dose consolidation. In addition, we did not observe differences in the duration of hospitalization, hematologic recovery, number of febrile episodes or of re-hospitalizations in myeloma patients aged 66-69 years compared to patients below 66 years. Therefore, our data suggest that $200 \mathrm{mg} / \mathrm{m} 2$ melphalan can be safely applied by an experienced transplant team to myeloma patients between 66-69 years within an experienced transplant team.

We applied a reduced dose of $140 \mathrm{mg} / \mathrm{m} 2$ melphalan to myeloma patients $\geq 70$ years in this study. We observed few, but notable differences compared to $200 \mathrm{mg} / \mathrm{m} 2$ melphalan in patients below 70 years. The duration of hospitalization was significantly longer in patients $\geq 70$ years ( 26 vs. 20 days), and the time until platelet (but not neutrophil) recovery above $20 \mathrm{G} / \mathrm{L}$ was longer (19 vs. 15 days). Since we did not observe more admissions to the intensive care unit, febrile episodes, or bleeding events, we concluded that the prolonged hospitalization of patients $\geq 70$ years was related to a generally slower recovery from the deterioration of the general condition associated with high-dose chemotherapy.

We observed similar rates of complete remission 100 days after ASCT in patients below 66 years and patients $\geq 66$ years. Also, the progression-free survival and the overall survival was not different between the three age groups in this study. Thus, 
these results suggest that elderly myeloma patients may equally benefit from consolidation treatment with HDCT and ASCT as younger myeloma patients in the era of novel agents. However, the obvious limitations of this study need to be stated including the retrospective, non-randomized, single-center design and the limited number of patients in the two elderly myeloma subgroups, as well as the limited follow-up.

\section{CONCLUSION}

Our data suggest that consolidation with HDCT and ASCT is feasible, safe and effective in myeloma patients older than 65 years. Age above 65 years, but also age above 70 years should not per se exclude patients from treatment with HDCT and ASCT. Therefore, the consolidation with HDCT and ASCT should be considered in elderly fit myeloma patients based on performance status and presence of complications and/or comorbidities, but not on chronological age alone. The sequential approach of $\mathrm{MM}$ treatment including ASCT can be challenging, but represents a feasible approach to further improve the outcome of elderly patients with multiple myeloma.

\section{ACKNOWLEDGEMENTS}

The authors wish to thank Irene Briner, Marion Bleckmann, Barbara Muster and Gaby Fahrni for help with data collection, and the apheresis, flow cytometry and stem cell laboratory teams for documentation of data relevant for this study 


\section{FIGURE LEGEND}

Figure 1: Depicted are Kaplan-Meier survival curves for the entire cohort of all 298 multiple myeloma patients for (A) progression free survival and (B) overall survival, as well as according to the three age groups for (C) progression free survival and (D) overall survival. 


\section{REFERENCES}

[1] Kyle RA, Child JA, Anderson $\mathrm{K}$ et al. Criteria for the classification of monoclonal gammopathies, multiple myeloma and related disorders: a report of the International Myeloma Working Group. British Journal of Haematology 2003; 121(5), 749-757.

[2] Palumbo A, Anderson K. Multiple myeloma. The New England Journal of Medicine 2011; 364(11):1046-1060.

[3] Alexanian R, Haut A, Khan AU et al. Treatment for multiple myeloma. Combination chemotherapy with different melphalan dose regimens. Journal of the American Medical Association 1969; 208(9): 1680-1685.

[4] Attal M, Harousseau JL, Stoppa AM et al. A prospective, randomized trial of autologous bone marrow transplantation and chemotherapy in multiple myeloma. Intergroupe Francais du Myelome. The New England Journal of Medicine 1996; 335(2): 91-97.

[5] Child JA, Morgan GJ, Davies FE et al. High-dose chemotherapy with hematopoietic stem-cell rescue for multiple myeloma. The New England Journal of Medicine 2003; 348(19):1875-1883.

[6] Harousseau JL, Attal M, Avet-Loiseau $\mathrm{H}$ et al. Bortezomib plus dexamethasone is superior to vincristine plus doxorubicin plus dexamethasone as induction treatment prior to autologous stem-cell transplantation in newly diagnosed multiple myeloma: results of the IFM 2005-01 phase III trial. Journal of Clinical Oncology 2010; 28(30): 4621-4629.

[7] Cavo M, Tacchetti P, Patriarca F et al. Bortezomib with thalidomide plus dexamethasone compared with thalidomide plus dexamethasone as induction 
therapy before, and consolidation therapy after, double autologous stem-cell transplantation in newly diagnosed multiple myeloma: a randomized phase 3 study. The Lancet 2010; 376(9758): 2075-2085.

[8] Sonneveld P, Schmidt-Wolf IG, van der Holt B et al. Bortezomib induction and maintenance treatment in patients with newly diagnosed multiple myeloma: results of the randomized phase III HOVON-65/ GMMG-HD4 trial. Journal of Clinical Oncology 2012; 30(24): 2946-2955.

[9] Sonneveld P, Goldschmidt H, Rosinol L et al. Bortezomib based versus nonbortezomib-based induction treatment before autologous stem-cell transplantation in patients with previously untreated multiple myeloma: a metaanalysis of phase III randomized, controlled trials. Journal of Clinical Oncology 2013; 31(26): 3279-3287.

[10] San Miguel JF, Schlag R, Khuageva NK et al. Bortezomib plus melphalan and prednisone for initial treatment of multiple myeloma. The New England Journal of Medicine 2008; 359(9): 906-917.

[11] Palumbo A, Bringhen $S$, Caravita $T$ et al. Oral melphalan and prednisone chemotherapy plus thalidomide compared with melphalan and prednisone alone in elderly patients with multiple myeloma: randomized controlled trial. The Lancet 2006; 367(9513): 825-831.

[12] Rajkumar SV, Jacobus S, Callander NS et al. Lenalidomide plus high-dose dexamethasone versus lenalidomide plus low dose dexamethasone as initial therapy for newly diagnosed multiple myeloma: an open-label randomized controlled trial. The Lancet Oncology 2010; 11(1): 29-37. 
[13] Martinez-Lopez J, Blade J, Mateos MV et al. Long-term prognostic significance of response in multiple myeloma after stem cell transplantation. Blood 2011; 118(3), 529-534.

[14] Gay F, Larocca A, Wijermans P et al. Complete response correlates with longterm progression-free and overall survival in elderly myeloma treated with novel agents: analysis of 1175 patients. Blood 2011; 117(11): 3025-3031.

[15] Brenner H, Gondos A, Pulte D. Recent major improvement in long-term survival of younger patients with multiple myeloma. Blood 2008; 111(5): 2521-2526.

[16] Pulte D, Gondos A, Brenner H. Improvement in survival of older adults with multiple myeloma: results of an updated period analysis of SEER data. Oncologist 2011; 16(11): 1600-1603.

[17] Pozzi S, Marcheselli L, Bari A et al. Survival of multiple myeloma patients in the era of novel therapies confirms the improvement in patients younger than 75 years: a population based analysis. British Journal of Haematology 2013; 163(1): 40-46.

[18] Palumbo A, Bringhen S, Ludwig $\mathrm{H}$ et al.Personalized therapy in multiple myeloma according to patient age and vulnerability: a report of the European Myeloma Network (EMN). Blood 2011; 118(17): 4519-4529.

[19] Badros A, Barlogie B, Siegel E et al. Autologous stem cell transplantation in elderly multiple myeloma patients over the age of 70 years. British Journal of Haematology 2001; 114(3): 600-607.

[20] Palumbo A, Triolo S, Argentino C et al. Dose-intensive melphalan with stem cell support (MEL 100) is superior to standard treatment in elderly myeloma patients. Blood 1999; 94(4): 1248-1253. 
[21] Klepin HD, Hurd DD. Autologous transplantation in elderly patients with multiple myeloma: are we asking the right questions? Bone Marrow Transplantation 2006; 38(9): 585-592.

[22] Mehta J, Cavo M, Singhal S. How I treat elderly patients with myeloma. Blood 2010; 116(13): 2215-2223.

[23] Siegel DS, Desikan KR, Mehta $\mathrm{J}$ et al. Age is not a prognostic variable with autotransplants for multiple myeloma. Blood 1999; 93(1): 51-54.

[24] Sirohi B, Powles R, Treleaven J et al. The role of autologous transplantation in patients with multiple myeloma aged 65 years and over. Bone Marrow Transplantation 2000; 25(5): 533-539.

[25] Reece DE, Bredeson C, Perez WS et al. Autologous stem cell transplantation in multiple myeloma patients $<60$ vs $\geq 60$ years of age. Bone Marrow Transplantation 2003; 32(12): 1135-1143.

[26] Jantunen E, Kuittinen T, Penttila K, Lehtonen P, Mahlamaki E, Nousiainen T. High-dose melphalan $(200 \mathrm{mg} / \mathrm{m} 2)$ supported by autologous stem cell transplantation is safe and effective in elderly ( $\geq 65$ years) myeloma patients: comparison with younger patients treated on the same protocol. Bone Marrow Transplantation 2006; 37(10): 917-922.

[27] Gertz MA, Lacy MQ, Dispenzieri A et al. Impact of age and serum creatinine value on outcome after autologous blood stem cell transplantation for patients with multiple myeloma. Bone Marrow Transplantation 2007; 39(10): 605-611.

[28] Kumar SK, Dingli D, Lacy MQ et al. Autologous stem cell transplantation in patients of 70 years and older with multiple myeloma: results from a matched pair analysis. The American Journal of Hematology 2008; 83(8): 614-617. 
[29] El Cheikh J, Kfoury E, Calmels B et al. Age at transplantation and outcome after autologous stem cell transplantation in elderly patients with multiple myeloma. Hematology/Oncology and Stem Cell Therapy 2001; 4(1): 30-36.

[30] Muta T, Miyamoto T, Fujisaki T et al. Evaluation of the feasibility and efficacy of autologous stem cell transplantation in elderly patients with multiple myeloma. Internal Medicine 2013; 52(1): 63-70.

[31] Bashir Q, Shah N, Parmar S et al. Feasibility of autologous hematopoietic stem cell transplant in patients aged $\geq 70$ years with multiple myeloma. Leukemia and Lymphoma 2012; 53(1): 118-122.

[32] Schmid A, Friess D, Mansouri Taleghani B et al. Role of plerixafor in autologous stem cell mobilization with vinorelbine chemotherapy and granulocyte-colony stimulating factor in patients with myeloma: a phase II study (PAV-trial). Leuk Lymphoma 2015; 56(3): 608-614.

[33] Keller S, Seipel K, Novak U et al. Neurotoxicity of stem cell mobilization chemotherapy with vinorelbine in myeloma patients after bortezomib treatment. Leuk Res 2015; 39(7): 786-792.

[34] Mueller BU, Keller S, Seipel K et al. Stem cell mobilization chemotherapy with gemcitabine is effective and safe in myeloma patients with bortezomib-induced neurotoxicity. Leuk Lymphoma 2016; 57(5): 1122-1129.

[35] Palumbo A, Gay F, Falco P et al. Bortezomib as induction before autologous transplantation, followed by lenalidomide as consolidation-maintenance in untreated multiple myeloma patients. Journal of Clinical Oncology 2010; 28(5): 800-807.

[36] Gay F, Magarotto V, Crippa C et al. Bortezomib induction, reduced-intensity transplantation, and lenalidomide consolidation-maintenance for myeloma: updated results. Blood 2013; 122(8): 1376-1383. 
[37] Straka C, Schaefer-Eckart K, Bassermann F et al. Prospective randomized trial of Len/Dex induction followed by tandem MEL 140 with autologous blood stem cell transplantation and Len maintenance versus continued therapy with Len/Dex in myeloma patients age 60-75 years: protocol-defined safety analysis after 100 patients. Blood 2012; 120(21): 2012.

[38] Kumar SK, Dispenzieri A, Lacy MQ et al. Continued improvement in survival in multiple myeloma: changes in early mortality and outcomes in older patients. Leukemia 2013; 28(5): 1122-1128

[39] Ozaki S, Harada T, Saitoh T et al. Survival of multiple myeloma patients aged 65-70 years in the era of novel agents and autologous stem cell transplantation: a multicenter retrospective collaborative study of the Japanese Society of Myeloma and the European Myeloma Network, Acta Haematologica 2014; 132(2): 211-219. 


\section{Figure 1}

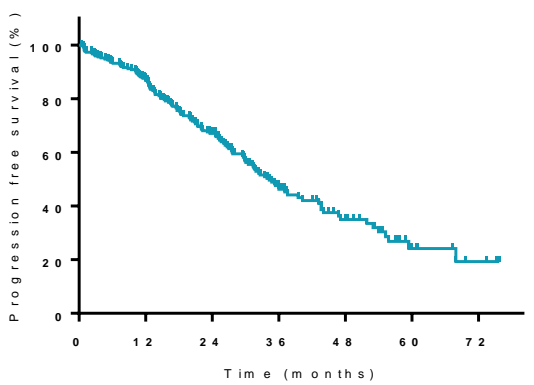

C

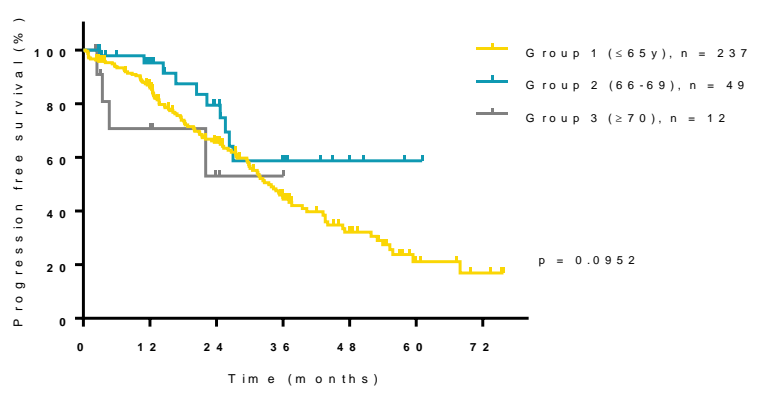

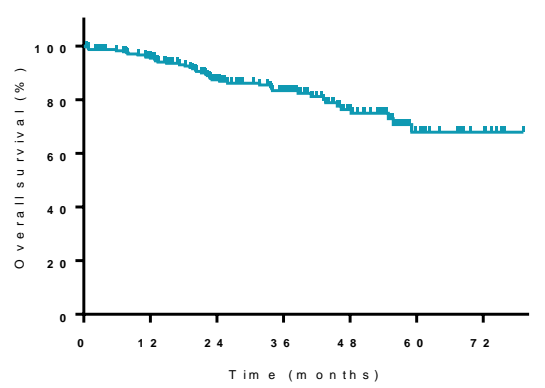

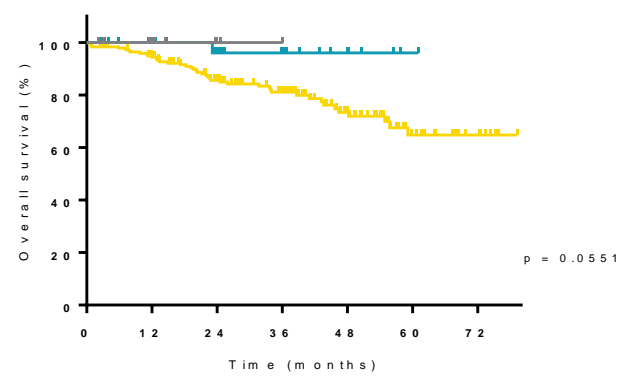

